\title{
Gauge Fixing the Standard Model Effective Field Theory
}

\author{
Andreas Helset, ${ }^{1, *}$ Michael Paraskevas, ${ }^{2, \dagger}$ and Michael Trott ${ }^{1, \dagger}$ \\ ${ }^{1}$ Niels Bohr International Academy and Discovery Centre, Niels Bohr Institute, University of Copenhagen, \\ Blegdamsvej 17, DK-2100 Copenhagen, Denmark \\ ${ }^{2}$ Department of Physics, University of Ioannina, GR 45110, Ioannina, Greece
}

(Received 3 April 2018; published 22 June 2018)

\begin{abstract}
We gauge-fix the standard model effective field theory in a manner invariant under background-field gauge transformations using a geometric description of the field connections.
\end{abstract}

DOI: 10.1103/PhysRevLett.120.251801

Introduction.-When physics beyond the standard model $(\mathrm{SM})$ is present at scales $(\Lambda)$ larger than the electroweak scale $\left.\left(\sqrt{2\left\langle H^{\dagger} H\right.}\right\rangle \equiv \bar{v}_{T}\right)$, the $\mathrm{SM}$ can be extended into an effective field theory (EFT). The standard model effective field theory (SMEFT), defined by a powercounting expansion in the ratio of scales $\bar{v}_{T} / \Lambda<1$, extends the SM with higher-dimensional operators $\mathcal{Q}_{i}^{(d)}$ of mass dimension $d$. The Lagrangian is

$$
\begin{aligned}
\mathcal{L}_{\mathrm{SMEFT}} & =\mathcal{L}_{\mathrm{SM}}+\mathcal{L}^{(5)}+\mathcal{L}^{(6)}+\mathcal{L}^{(7)}+\cdots, \\
\mathcal{L}^{(d)} & =\sum_{i} \frac{C_{i}^{(d)}}{\Lambda^{d-4}} \mathcal{Q}_{i}^{(d)} \quad \text { for } d>4 .
\end{aligned}
$$

The SMEFT is a model-independent and consistent lowenergy parametrization of heavy physics beyond the SM, so long as its defining assumptions are satisfied: that there are no light hidden states in the spectrum with couplings to the $\mathrm{SM}$, and a $S U(2)_{L}$ scalar doublet with hypercharge $\mathrm{y}_{h}=1 / 2$ is present in the EFT.

The SMEFT has the same $S U(3)_{C} \times S U(2)_{L} \times U(1)_{Y}$ global symmetry as the SM. The SMEFT also has a phase where $S U(2)_{L} \times U(1)_{Y} \rightarrow U(1)_{\mathrm{em}}$ due to the Higgs mechanism. A difference between these theories is that additional couplings and interactions between the fields come about due to the $\mathcal{Q}_{i}^{(d)}$. Some of these interactions are bilinear in the SM fields in the phase broken by the Higgs mechanism. These terms are important for gauge fixing, and the presence of these interactions introduces technical challenges to the usual gauge-fixing approach.

The bilinear field interactions in the SMEFT are usefully thought of in terms of connections in the field space manifolds of the theory $[1,2]$. The purpose of this Letter is to show that gauge-fixing the SMEFT, taking into

Published by the American Physical Society under the terms of the Creative Commons Attribution 4.0 International license. Further distribution of this work must maintain attribution to the author(s) and the published article's title, journal citation, and DOI. Funded by SCOAP. account the field space metrics, directly resolves many of the technical challenges that have been identified to date. The approach we develop generalizes directly to higher orders in the SMEFT power-counting expansion.

The difficulties in gauge-fixing the SMEFT are also present when the background-field method (BFM) [3-9] is used [10]. The BFM splits the fields in the theory into quantum and classical fields $(F \rightarrow F+\hat{F})$, with the latter denoted with a hat superscript. One performs a gaugefixing procedure that preserves background-field gauge invariance while breaking explicitly the quantum-field gauge invariance. This allows a gauge choice for the quantum fields to be made to one's advantage, while still benefiting from the simplifications that result from naive Ward identities [11] due to the preserved background-field gauge invariance. (The Ward identities result from considering BRST invariance [12] when the BFM is not used, which can be more cumbersome when extending results to higher orders in the SMEFT power-counting expansion.)

In this Letter, we show how to perform gauge fixing with the BFM, taking into account the field space metrics that are present due to the SMEFT power-counting expansion. The usual $R_{\xi}$ gauge-fixing approach in the BFM for the standard model [6-9] is a special case of this approach. (For an $R_{\xi}$ gauge SMEFT formulation with three distinct $\xi$ parameters, see Ref. [13].) Conceptually, one can understand that this procedure is advantageous, as it preserves the background $S U(3)_{C} \times S U(2)_{L} \times U(1)_{Y}$ invariance on the curved field spaces present due to the power-counting expansion. The latter is trivialized away in the standard model.

Scalar space.-The operators that lead to scalar kinetic terms in the phase of the theory up to $\mathcal{L}^{(6)}$ broken by the Higgs mechanism are [14]

$$
\begin{aligned}
\mathcal{L}_{\text {scalar,kin }}= & \left(D_{\mu} H\right)^{\dagger}\left(D^{\mu} H\right)+\frac{C_{\mathrm{H} \square}}{\Lambda^{2}}\left(H^{\dagger} H\right) \square\left(H^{\dagger} H\right) \\
& +\frac{C_{\mathrm{HD}}}{\Lambda^{2}}\left(H^{\dagger} D_{\mu} H\right)^{*}\left(H^{\dagger} D^{\mu} H\right), \\
\equiv & \frac{1}{2} h_{I J}(\phi)\left(D_{\mu} \phi\right)^{I}\left(D^{\mu} \phi\right)^{J} .
\end{aligned}
$$


Our covariant derivative sign convention is given by $D^{\mu} H=$ $\left(\partial^{\mu}+i g_{2} W^{a, \mu} \sigma_{a} / 2+i g_{1} \mathrm{y}_{h} B^{\mu} Y\right) H$ and $\left(D^{\mu} \phi\right)^{I}=\left(\partial^{\mu} \delta_{J}^{I}-\right.$ $\left.\frac{1}{2} W^{A, \mu} \tilde{\gamma}_{A, J}^{I}\right) \phi^{J}$, with definitions given below. Defining

$$
H=\frac{1}{\sqrt{2}}\left[\begin{array}{l}
\phi_{2}+i \phi_{1} \\
\phi_{4}-i \phi_{3}
\end{array}\right]
$$

the scalar field connections can be described by an $\mathbb{R}^{4}$ field manifold with the metric $h_{I J}(\phi)$. Our notation is that the latin capital letters $I, J, K, L, \ldots$ run over $\{1,2,3,4\}$, while lowercase latin letters $i, j, k, l, \ldots$ run over $\{1,2\}$. The metric takes the form

$$
h_{I J}(\phi)=\delta_{I J}-2 \frac{C_{\mathrm{H} \square}}{\Lambda^{2}} \phi_{I} \phi_{J}+\frac{1}{2} \frac{C_{\mathrm{HD}}}{\Lambda^{2}} f_{I J}(\phi),
$$

where

$$
f_{I J}(\phi)=\left[\begin{array}{cccc}
a & 0 & d & c \\
0 & a & c & -d \\
d & c & b & 0 \\
c & -d & 0 & b
\end{array}\right], \quad \begin{aligned}
& a=\phi_{1}^{2}+\phi_{2}^{2}, \\
& b=\phi_{3}^{2}+\phi_{4}^{2}, \\
& c=\phi_{1} \phi_{4}+\phi_{2} \phi_{3}, \\
& d=\phi_{1} \phi_{3}-\phi_{2} \phi_{4} .
\end{aligned}
$$

The Riemann curvature tensor calculated from the scalar field metric is nonvanishing $[1,2,15]$. The scalar manifold is curved due to the power-counting expansion. An interesting consequence is that there does not exist a gauge-independent field redefinition which sets $h_{I J}=$ $\delta_{I J}$ when considering $\mathcal{L}^{(6)}$ corrections [15]. As a result, demanding that the Higgs doublet field be canonically normalized in the SMEFT to $\mathcal{L}^{(6)}$ cannot be used as a defining condition for operator bases [15-18].

Gauge boson space.-The operators that lead to $C P$-even bilinear interactions for the $S U(2)_{L} \times U(1)_{Y}$ spin-1 fields up to $\mathcal{L}^{(6)}$ are

$$
\begin{aligned}
\mathcal{L}_{\mathrm{WB}}= & -\frac{1}{4} W_{\mu \nu}^{a} W^{a, \mu \nu}-\frac{1}{4} B_{\mu \nu} B^{\mu \nu}+\frac{C_{\mathrm{HB}}}{\Lambda^{2}} H^{\dagger} H B_{\mu \nu} B^{\mu \nu} \\
& +\frac{C_{\mathrm{HW}}}{\Lambda^{2}} H^{\dagger} H W_{\mu \nu}^{a} W^{a, \mu \nu}+\frac{C_{\mathrm{HWB}}}{\Lambda^{2}} H^{\dagger} \sigma^{a} H W_{\mu \nu}^{a} B^{\mu \nu}, \\
\equiv & -\frac{1}{4} g_{A B}(H) \mathcal{W}_{\mu \nu}^{A} \mathcal{W}^{B, \mu \nu},
\end{aligned}
$$

where $a, b, \ldots$ run over $\{1,2,3\}$, and $A, B, C, \ldots$ run over $\{1,2,3,4\}$. Here $\mathcal{W}_{\mu \nu}^{4}=B_{\mu \nu}$. Analogous to the scalar sector, we have introduced a metric $g_{A B}\left[H\left(\phi_{i}\right)\right]$, taking the form $g_{a b}=\left(1-4 \frac{C_{\mathrm{HW}}}{\Lambda^{2}} H^{\dagger} H\right) \delta_{a b}, \quad g_{44}=1-4 \frac{C_{\mathrm{HB}}}{\Lambda^{2}} H^{\dagger} H$,

$g_{a 4}=g_{4 a}=-2 \frac{C_{\mathrm{HWB}}}{\Lambda^{2}} H^{\dagger} \sigma_{a} H$.

The Riemann curvature tensor for the gauge fields can be calculated from $g_{A B}$ and is nonvanishing; the $(C P$-even $) \mathbb{R}^{4}$ spin-1 field manifold is also curved. $\left[S U(2)_{L}\right.$ is self adjoint. As a result, one can define a $\mathbb{G}^{A B}$ tensor of the same form as $g_{A B}$ through $\mathbb{G}^{A B}(H) \mathcal{W}_{A}^{\mu \nu} \mathcal{W}_{B, \mu \nu}$. This $\mathbb{G}^{A B}$ is not the tensor $g^{A B}$ defined through the relation $g^{A B} g_{B C}=\delta_{C}^{A}$ and used in the gauge-fixing term.] A physical consequence is that, as in the case of the scalar manifold, there does not exist a gaugeindependent field redefinition that sets $g_{A B}=\delta_{A B}$ including $\mathcal{L}^{(6)}$ corrections. (A rotation to the mass eigenstate basis for the field bilinear interactions can be made, and this is consistent with the curvature of the gauge manifold. Fieldredefiniton-invariant quantities are more directly connected to $S$-matrix elements. For a similar discussion of how fieldredefintion-invariant beta functions can be defined in the SMEFT, see Ref. [19].) The power-counting expansion of the SMEFT is relevant for gauge fixing and cannot be removed with gauge-independent field redefinitions, which is a novel feature compared to more familiar EFTs without a phase broken by the Higgs mechanism. The particular form of the field space metrics depends on the operator basis used, but the utility of the geometric approach developed here does not. This argues for a modified gauge-fixing procedure using the BFM in the SMEFT.

Gauge fixing.-Eliminating bilinear kinetic mixing between the gauge bosons and the Goldstone bosons in an efficient gauge-fixing procedure is advantageous. A simpler LSZ procedure [20] to construct $S$-matrix elements results from this condition being imposed. $R_{\xi}$ gauge [21] in the SM when $\xi_{W}=\xi_{B}$ has some further advantages in eliminating contact operators that complicate calculations in intermediate steps. Using the BFM combined with $R_{\xi}$ gauge fixing, the gauge-fixing term for the $S U(2)_{L} \times$ $U(1)_{Y}$ fields in the SM takes the form [6-9]

$$
\begin{aligned}
\mathcal{L}_{\mathrm{GF}}= & -\frac{1}{2 \xi_{W}} \sum_{a}\left[\partial_{\mu} W^{a, \mu}-g_{2} \epsilon^{a b c} \hat{W}_{b, \mu} W_{c}^{\mu}\right. \\
& \left.+i g_{2} \frac{\xi_{W}}{2}\left(\hat{H}_{i}^{\dagger}\left(\sigma^{a}\right)_{j}^{i} H^{j}-H_{i}^{\dagger}\left(\sigma^{a}\right)_{j}^{i} \hat{H}^{j}\right)\right]^{2} \\
& -\frac{1}{2 \xi_{B}}\left[\partial_{\mu} B^{\mu}+i g_{1} \frac{\xi_{B}}{2}\left(\hat{H}_{i}^{\dagger} H^{i}-H_{i}^{\dagger} \hat{H}^{i}\right)\right]^{2},
\end{aligned}
$$

where the background fields are denoted by a hat.

The $S U(2)_{L}$ Pauli matrix representation in Eq. (8) is inconvenient for characterizing the gauge-fixing term, as $g_{A B}$ is defined on $\mathbb{R}^{4}$. The Pauli matrix algebra is isomorphic to the Clifford algebra $C(0,3)$, and the latter can be embedded in the $\mathbb{R}^{4}$ field space using the real representations $\gamma_{1,2,3}$ such that 


$$
\begin{aligned}
& \gamma_{1, J}^{I}=\left[\begin{array}{cccc}
0 & 0 & 0 & -1 \\
0 & 0 & -1 & 0 \\
0 & 1 & 0 & 0 \\
1 & 0 & 0 & 0
\end{array}\right], \quad \gamma_{2, J}^{I}=\left[\begin{array}{cccc}
0 & 0 & 1 & 0 \\
0 & 0 & 0 & -1 \\
-1 & 0 & 0 & 0 \\
0 & 1 & 0 & 0
\end{array}\right], \\
& \gamma_{3, J}^{I}=\left[\begin{array}{cccc}
0 & -1 & 0 & 0 \\
1 & 0 & 0 & 0 \\
0 & 0 & 0 & -1 \\
0 & 0 & 1 & 0
\end{array}\right], \quad \gamma_{4, J}^{I}=\left[\begin{array}{cccc}
0 & -1 & 0 & 0 \\
1 & 0 & 0 & 0 \\
0 & 0 & 0 & 1 \\
0 & 0 & -1 & 0
\end{array}\right] \text {. }
\end{aligned}
$$

The $\gamma_{4}$ generator is used for the $U(1)_{Y}$ embedding. As $S U(2)_{L}$ is self-adjoint, we can also define this algebra for the adjoint fields, using the same real representations. $\gamma_{1,2,3,4}\langle\phi\rangle \neq 0$, and the unbroken combination of generators $\left(\gamma_{3}+\gamma_{4}\right)\langle\phi\rangle=0$ corresponds to $U(1)_{\mathrm{em}}$. We absorb the couplings into the structure constants and gamma matrices,

$$
\begin{gathered}
\tilde{\epsilon}_{B C}^{A}=g_{2} \epsilon_{B C}^{A}, \quad \text { with } \tilde{\epsilon}_{23}^{1}=+g_{2}, \\
\tilde{\gamma}_{A, J}^{I}= \begin{cases}g_{2} \gamma_{A, J}^{I}, & \text { for } A=1,2,3 \\
g_{1} \gamma_{A, J}^{I}, & \text { for } A=4 .\end{cases}
\end{gathered}
$$

The different couplings $g_{1}, g_{2}$ enter as the group defined on the $\mathbb{R}^{4}$ field space is not simple. The $\gamma_{a, J}^{I}$ matrices satisfy the algebra $\left[\tilde{\gamma}_{a}, \tilde{\gamma}_{b}\right]=2 \tilde{\epsilon}_{a b}^{c} \tilde{\gamma}_{c}$ and $\left[\tilde{\gamma}_{a}, \tilde{\gamma}_{4}\right]=0$. The structure constants vanish when any of $A, B$, $C=4$. Note also that $\hat{H}^{\dagger} \sigma_{A} H-H^{\dagger} \sigma_{A} \hat{H}=-i \phi \gamma_{A} \hat{\phi}$, with $\sigma_{4}=Y=\mathbb{I}_{2 \times 2}$. The gauge-fixing term in the backgroundfield gauge takes the form

$$
\begin{aligned}
\mathcal{L}_{\mathrm{GF}} & =-\frac{\hat{g}_{A B}}{2 \xi} \mathcal{G}^{A} \mathcal{G}^{B}, \\
\mathcal{G}^{X} & \equiv \partial_{\mu} \mathcal{W}^{X, \mu}-\tilde{\epsilon}_{C D}^{X} \hat{\mathcal{W}}_{\mu}^{C} \mathcal{W}^{D, \mu}+\frac{\xi}{2} \hat{g}^{X C} \phi^{I} \hat{h}_{I K} \tilde{\gamma}_{C, J}^{K} \hat{\phi}^{J} .
\end{aligned}
$$

The $R_{\xi}$ gauge-fixing term follows when replacing the background fields with their vacuum expectation values. The gauge-fixing term is bilinear in the quantum fields. The field space metrics in Eq. (11) are denoted with a hat superscript, indicating that they are defined to depend only on the background fields. Contracting with the field space metrics is a basis-independent feature of the gaugefixing term.

It is useful to note the following background-field gauge transformations $(\delta \hat{F})$, with infinitesimal local gauge parameters $\delta \hat{\alpha}_{A}(x)$ when verifying explicitly the background-field gauge invariance of this expression:

$$
\begin{aligned}
\delta \hat{\phi}^{I} & =-\delta \hat{\alpha}^{A} \frac{\tilde{\gamma}_{A, J}^{I}}{2} \hat{\phi}^{J}, \\
\delta\left(D^{\mu} \hat{\phi}\right)^{I} & =-\delta \hat{\alpha}^{A} \frac{\tilde{\gamma}_{A, J}^{I}}{2}\left(D^{\mu} \hat{\phi}\right)^{J}, \\
\delta \hat{\mathcal{W}}^{A, \mu} & =-\partial^{\mu}\left(\delta \hat{\alpha}^{A}\right)-\tilde{\epsilon}^{A}{ }_{B C} \delta \hat{\alpha}^{B} \hat{\mathcal{W}}^{C, \mu}, \\
\delta \hat{h}_{I J} & =\hat{h}_{K J} \frac{\delta \hat{\alpha}^{A} \tilde{\gamma}_{A, I}^{K}}{2}+\hat{h}_{I K} \frac{\delta \hat{\alpha}^{A} \tilde{\gamma}_{A, J}^{K}}{2}, \\
\delta \hat{\mathcal{W}}_{\mu \nu}^{A} & =-\tilde{\epsilon}_{B C}^{A} \delta \hat{\alpha}^{B} \hat{\mathcal{W}}_{\mu \nu}^{C}, \\
\delta \hat{g}_{A B} & =\hat{g}_{C B} \tilde{\epsilon}^{C}{ }_{D A} \delta \hat{\alpha}^{D}+\hat{g}_{A C} \tilde{\epsilon}^{C}{ }_{D B} \delta \hat{\alpha}^{D} .
\end{aligned}
$$

The background-field gauge invariance is established by using these transformations in conjuction with a linear change of variables on the quantum fields

$$
\begin{aligned}
\mathcal{W}^{A, \mu} & \rightarrow \mathcal{W}^{A, \mu}-\tilde{\epsilon}_{B C}^{A} \delta \hat{\alpha}^{B} \mathcal{W}^{C, \mu}, \\
\phi^{I} & \rightarrow \phi^{I}-\frac{\delta \hat{\alpha}^{B} \tilde{\gamma}_{B, K}^{I}}{2} \phi^{K} .
\end{aligned}
$$

The transformation of the gauge-fixing term is

$$
\delta \mathcal{G}^{X}=-\tilde{\epsilon}^{X}{ }_{A B} \delta \hat{\alpha}^{A} \mathcal{G}^{B} .
$$

With these transformations, the background-field gauge invariance of the gauge-fixing term is directly established.

The background-field-generating functional $(Z)$ depends on the background fields $\hat{F} \equiv\left\{\hat{\mathcal{W}}^{A}, \hat{\phi}^{I}\right\}$ and the sources $J_{F} \equiv\left\{J^{A}, J_{\phi}^{I}\right\}$. The source terms transform as

$$
\delta J_{\mu}^{A}=-\tilde{\epsilon}_{B C}^{A} \delta \hat{\alpha}^{B} J_{\mu}^{C}, \quad \delta J_{\phi}^{I}=-\frac{\delta \hat{\alpha}^{B} \tilde{\gamma}_{B, K}^{I}}{2} J_{\phi}^{K} .
$$

The background-field-generating functional dependence on the source terms is invariant under the background-field gauge transformations, as they are contracted with the field space metrics in $Z\left[\hat{F}, J_{F}\right]$ defined by

$$
\int \mathcal{D} F \operatorname{det}\left[\frac{\Delta \mathcal{G}^{A}}{\Delta \alpha^{B}}\right] e^{i\left(S[F+\hat{F}]+\mathcal{L}_{\mathrm{GF}}+\hat{g}_{C D} J_{\mu}^{C} \mathcal{W}^{D, \mu}+\hat{h}_{I J} J_{\phi}^{I} \phi^{J}\right)} .
$$

The integration over $d x^{4}$ is implicit in this expression. Here a quantum-field gauge transformation is indicated with a $\Delta$. The action is manifestly invariant under the gauge transformation of $F+\hat{F}$. This establishes the background field invariance of the generating functional.

The quantum-field gauge transformations are

$$
\begin{aligned}
\Delta \mathcal{W}_{\mu}^{A} & =-\partial_{\mu} \Delta \alpha^{A}-\tilde{\epsilon}_{B C}^{A} \Delta \alpha^{B}\left(\mathcal{W}_{\mu}^{C}+\hat{\mathcal{W}}_{\mu}^{C}\right), \\
\Delta \phi^{I} & =-\Delta \alpha^{A} \frac{\tilde{\gamma}_{A, J}^{I}}{2}\left(\phi^{J}+\hat{\phi}^{J}\right) .
\end{aligned}
$$

As the field metrics in Eq. (11) depend only on the background fields and do not transform under quantum-field 
gauge transformations, the Faddeev-Popov [22] ghost term still follows directly; we find

$$
\begin{aligned}
\mathcal{L}_{\mathrm{FP}}= & -\hat{g}_{A B} \bar{u}^{B}\left[-\partial^{2} \delta_{C}^{A}-\overleftarrow{\partial}_{\mu} \tilde{\epsilon}^{A}{ }_{D C}\left(\mathcal{W}^{D, \mu}+\hat{\mathcal{W}}^{D, \mu}\right)\right. \\
& +\tilde{\epsilon}^{A}{ }_{D C} \hat{\mathcal{W}}_{\mu}^{D} \vec{\partial}^{\mu}-\tilde{\epsilon}^{A}{ }_{D E} \tilde{\epsilon}^{E}{ }_{F C} \hat{\mathcal{W}}_{\mu}^{D}\left(\mathcal{W}^{F, \mu}+\hat{\mathcal{W}}^{F, \mu}\right) \\
& \left.\left.-\frac{\xi}{4} \hat{g}^{A D}\left(\phi^{J}+\hat{\phi}^{J}\right) \tilde{\gamma}_{C, J}^{I} \hat{h}_{I K} \tilde{\gamma}_{D, L}^{K} \hat{\phi}^{L}\right)\right] u^{C} .
\end{aligned}
$$

The form of this expression follows from the convention choice in Eq. (6), and the descendent convention in Eq. (11). The mass eigenstate $\mathcal{Z}_{\mu}, \mathcal{A}_{\mu}$ fields are defined by

$$
\left[\begin{array}{c}
W_{\mu}^{3} \\
B_{\mu}
\end{array}\right]=\left[\begin{array}{cc}
1+\frac{C_{\mathrm{HW}} \bar{v}_{T}^{2}}{\Lambda^{2}} & -\frac{C_{\mathrm{HWB}} \bar{v}_{T}^{2}}{2 \Lambda^{2}} \\
-\frac{C_{\mathrm{HWB}} \bar{v}_{T}^{2}}{2 \Lambda^{2}} & 1+\frac{C_{\mathrm{HB}} \bar{v}_{T}^{2}}{\Lambda^{2}}
\end{array}\right]\left[\begin{array}{cc}
c_{\bar{\theta}} & s_{\bar{\theta}} \\
-s_{\bar{\theta}} & c_{\bar{\theta}}
\end{array}\right]\left[\begin{array}{c}
\mathcal{Z}_{\mu} \\
\mathcal{A}_{\mu}
\end{array}\right],
$$

where the introduced rotation angles $s_{\bar{\theta}}, c_{\bar{\theta}}$ are $[23,24]$

$$
t_{\bar{\theta}} \equiv \frac{s_{\bar{\theta}}}{c_{\bar{\theta}}}=\frac{\bar{g}_{1}}{\bar{g}_{2}}+\frac{\bar{v}_{T}^{2}}{2} \frac{C_{\mathrm{HWB}}}{\Lambda^{2}}\left(1-\frac{\bar{g}_{1}^{2}}{\bar{g}_{2}^{2}}\right),
$$

and $\bar{g}_{2}=g_{2}\left(1+C_{\mathrm{HW}} \bar{v}_{T}^{2} / \Lambda^{2}\right), \quad \bar{g}_{1}=g_{1}\left(1+C_{\mathrm{HB}} \bar{v}_{T}^{2} / \Lambda^{2}\right)$. This removes mixing terms as well as making the kinetic term of the spin-1 electroweak fields canonically normalized. This results in a simplified LSZ procedure to construct $S$-matrix elements. Ghost fields associated with the mass eigenstates follow from the linear rotation to the mass eigenstate fields. Feynman rules can be extracted directly from these expressions. Corrections from the higher-dimensional operators $\left(C_{\mathrm{H}}, C_{\mathrm{HD}}, C_{\mathrm{HWB}}, C_{\mathrm{HB}}, C_{\mathrm{HW}}\right)$ enter in ghost interactions and couple to the sources through the gauge and scalar metrics.

Conclusions. - In this Letter we have defined an approach to gauge-fixing the SMEFT that preserves background-field gauge invariance. This approach directly generalizes to higher orders in the SMEFT power counting. The key point is to gauge-fix the fields on the curved field space due to the power-counting expansion.

M. T. and A. H. acknowledge support from the Villum Fonden and the Danish National Research Foundation (DNRF91) through the Discovery center. M. T. is grateful to the Mainz Institute for Theoretical Physics (MITP) for hospitality and partial support during the completion of this work. We thank E. Bjerrum-Bohr, I. Brivio, P. Damgaard, C. Hartmann, A. Manohar, and G. Passarino for useful discussions related to this material and comments on the draft.

*ahelset@nbi.ku.dk mparask@grads.uoi.gr *michael.trott@cern.ch

[1] R. Alonso, E. E. Jenkins, and A. V. Manohar, Phys. Lett. B 754, 335 (2016).

[2] R. Alonso, E. E. Jenkins, and A. V. Manohar, J. High Energy Phys. 08 (2016) 101.

[3] B. S. DeWitt, Phys. Rev. 162, 1195 (1967).

[4] G. 't Hooft, Nucl. Phys. B62, 444 (1973).

[5] L. F. Abbott, Acta Phys. Pol. B 13, 33 (1982).

[6] G. M. Shore, Ann. Phys. (N.Y.) 137, 262 (1981).

[7] M. B. Einhorn and J. Wudka, Phys. Rev. D 39, 2758 (1989).

[8] A. Denner, G. Weiglein, and S. Dittmaier, Phys. Lett. B 333, 420 (1994).

[9] A. Denner, G. Weiglein, and S. Dittmaier, Nucl. Phys. B440, 95 (1995).

[10] C. Hartmann and M. Trott, J. High Energy Phys. 07 (2015) 151.

[11] J. C. Ward, Phys. Rev. 78, 182 (1950).

[12] C. Becchi, A. Rouet, and R. Stora, Ann. Phys. (N.Y.) 98, 287 (1976).

[13] A. Dedes, W. Materkowska, M. Paraskevas, J. Rosiek, and K. Suxho, J. High Energy Phys. 06 (2017) 143.

[14] B. Grzadkowski, M. Iskrzynski, M. Misiak, and J. Rosiek, J. High Energy Phys. 10 (2010) 085.

[15] C. P. Burgess, H. M. Lee, and M. Trott, J. High Energy Phys. 07 (2010) 007.

[16] G. Passarino and M. Trott, arXiv: 1610.08356.

[17] G. Passarino, Eur. Phys. J. Plus 132, 16 (2017).

[18] I. Brivio and M. Trott, arXiv:1706.08945.

[19] M. B. Einhorn and J. Wudka, J. High Energy Phys. 08 (2001) 025.

[20] H. Lehmann, K. Symanzik, and W. Zimmermann, Nuovo Cimento 1, 205 (1955).

[21] G. 't Hooft and M. J. G. Veltman, Nucl. Phys. B50, 318 (1972).

[22] L. D. Faddeev and V. N. Popov, Phys. Lett. 25B, 29 (1967).

[23] B. Grinstein and M. B. Wise, Phys. Lett. B 265, 326 (1991).

[24] R. Alonso, E. E. Jenkins, A. V. Manohar, and M. Trott, J. High Energy Phys. 04 (2014) 159. 